\title{
FUNDAMENTAL EQUATION OF STATE OF ARGON, SATISFYING THE SCALING HYPOTHESIS AND WORKING IN THE REGION OF HIGH TEMPERATURES AND PRESSURES
}

\author{
S. V. RYKOV ${ }^{1^{\star}}$, V. A. RYKOV ${ }^{1}$, I. V. KUDRYAVTSEVA ${ }^{1}$, E. E. USTYUZHANIN ${ }^{2}$, \\ A. V.SVERDLOV ${ }^{1}$ \\ ${ }^{1}$ ITMO University \\ Kronvergskiy 49, 197101 Saint-Petersburg, Russia \\ 2 National Research University Moscow Power Engineering Institute \\ Krasnokazarmennaya 14, 111250 Moscow, Russia \\ * Corresponding author. E-mail: togg1@yandex.ru
}

DOI: $10.20948 /$ mathmontis-2020-47-11

Summary. This paper discusses the problem of describing thermodynamic properties of a substance at high temperatures and pressures on the basis of the fundamental equation of state (FEoS). This FEoS has the following characteristics: it transforms into the virial equation of state in the region of low densities; it is converted into the Berestov equation in the vicinity of the critical point. FEoS testing has been carried out on known thermodynamic properties of argon and has allowed establishing its workspace: by the pressure up to $1000 \mathrm{MPa}$; by the temperature from the temperature of the triple point to $1200 \mathrm{~K}$. It has been shown that our FEoS can qualitatively correctly describe the thermal surface of argon up to $17000 \mathrm{~K}$. A comparison of FEoS has been made with some well-known equations of state. When developing FEoS of argon, we have used elements of the similarity theory, which has allowed reducing the number of individual parameters of this FEoS.

\section{INTRODUCTION}

We investigate a problem of describing the thermophysical properties of substances in a wide range of temperatures and pressures including the critical region. The problem attracts the attention of many researchers [1-24]. In particular, this problem is actual when studying the behavior of substances:

- in the range of highly developed density fluctuations near the critical point;

- at high temperatures and high pressures.

To describe the properties of pure substances at high pressures and high temperatures, the authors of [25-32] have developed a number of fundamental equations of state (FEoS). When describing the liquid behavior in the vicinity of the critical point, we have used previously a number of approaches and developed some equations of state (EoS):

2010 Mathematics Subject Classification: 80A05, 80A10, 80M50.

Key words and phrases: mathematical modeling, thermodynamic surface, critical point, argon, equation of state. 
- scaling EoSs and crossover EoSs in a parametric form and with (the density, the temperature variables) $[1,2,10,12,16,24]$;

- FEoS of the virial type $[3,6,9,17]$;

- FEoS [14] based on requirements of the scaling theory (ST) for the critical region [33];

- FEoS $[4,5,7,8,11,15,18-20,22,23]$ converted into a Widom EoS and valid in the vicinity of the critical point.

We have analyzed approaches [1-12,14-20,22-24] and have got the following results. Scaling EoSs $[1,2,16,24]$ and crossover EoSs $[10,12]$ meet the requirements of ST [33], but they have a narrow work area limited by temperatures $T\left(0.9 T_{c}<T<2 T_{c}\right.$ [12], here $T_{c}$ is the critical temperature), and, therefore, can not be used when modeling thermodynamic properties of a substance in the range of high temperatures and high pressures.

One of the disadvantages of the crossover EoS [12] is the need to use different critical temperatures: one $T_{c}$ to calculate the pressure $(p)$ and another $T_{c}$ to calculate the isochoric heat capacity $\left(C_{V}\right)$. FEoS $[3,6,9,17]$ do not meet the requirements of ST. Therefore, these EFoSs do not describe the sound velocity $(w), C_{V}$, the isobar heat capacity $\left(C_{p}\right)$, and isothermal compressibility factor $(K)$ in the critical region with acceptably small uncertainties. At the same time, these EFoSs describe the equilibrium properties of argon in the regular part of the thermodynamic surface with low uncertainties. For example, FEoS of argon is proposed [9] in this form. The workspace of FEoS FEoS [9] is (limited by pressures $0 \leqslant p \leqslant 1000 \mathrm{MPa}$, by temperatures $83 \leqslant T \leqslant 700 \mathrm{~K}$ ) and can be successfully used when predicting thermal properties at high temperatures.

Bezverhiy et al [14] has developed FEoS, which takes into account the feature presence of $C_{V}(T, \rho)$ as a known function in the critical region, here $\rho$ is the density. Our analysis shows that EFoS [14] reproduces power laws of ST qualitatively incorrectly. For example, the critical isotherm [14] follows $\Delta p \propto(\Delta \rho)^{3}$. It should be $\Delta p \propto \Delta \rho|\Delta \rho|^{\delta-1}$ [33], here $\Delta p=\left(p-p_{c}\right) / p_{c}$; $\Delta \rho=\left(\rho-\rho_{c}\right) / \rho_{c} ; p_{c}$ is the critical pressure; $\rho_{c}$ is the critical density; $\delta$ is the critical index of the critical isotherm.

Empirical FeoSs are proposed in $[5,7,8,11]$. They qualitatively correctly reproduce all of ST power laws when describing properties in the vicinity of the critical point.

On the basis of the phenomenological theory of the critical point [34], the authors of [4, 15, 18-20, 22, 23] developed FEoS which is not inferior to scaling EoS and crossover EoS when describing the asymptotic vicinity of the critical point. We mark that FEoSs $[4,5,7,8$, $11,15,18-20,22,23]$ do not satisfy the theory of extended scaling [2]. Indeed, the function, $C_{V}\left(T, \rho_{c}\right)[4,5,7,8,11,14,15,18-20,22,23]$, follows $C_{V} \simeq A \tau^{-\alpha}+C \tau$. It is shown in [2] that 
$C_{V}\left(T, \rho_{c}\right)$ should be $C_{V} \simeq A \tau^{-\alpha}+B \tau^{-\alpha+\Delta}+C \tau$, here $\tau=\left(T-T_{c}\right) / T_{c} ; \alpha$ and $\Delta$ are the critical indexes. In addition, our analysis shows that FEoSs $[4,5,7,8,11,14,15,18-20,22,23]$ are inferior to FEoS [9] when describing properties at high temperature region. For example, there are a discrepancy between $p$ values calculated by EFoS [9] and $p$ values calculated by [20] at $17000 \mathrm{~K}$ these deviations exceed $50 \%$.

In this paper on the basis of the approach [35], we plan to develop a FEoS that meets the following requirements:

- satisfies ST requirements [33] and does not inferior to EoSs [10,12] when describing properties in the critical region;

- simulates the thermal surface of argon at temperatures up to $17000 \mathrm{~K}$ and by pressures up to $12 \mathrm{GPa}$;

- can be converted into the Berestov equation [2] in the critical region.

\section{STRUCTURE OF FEOS}

By analogy with $[4,15,18-20,22,23]$, this FEoS has the following structure:

$$
F(\rho, T)=F_{\text {reg }}(\rho, T)+F_{\text {nreg }}(\rho, T),
$$

where $F(\rho, T)$ is the Helmholtz free energy; $F_{\text {reg }}(\rho, T)$ is a regular function; $F_{\text {nreg }}(\rho, T)$ is an irregular component of the Helmholtz free energy:

$$
F_{\mathrm{nreg}}(\rho, T)=R T_{c} \phi(\omega, t)\left(|\Delta \rho|^{\delta+1} a_{0}(x)+|\Delta \rho|^{\delta+1+\frac{\Delta}{\beta}} a_{1}(x)\right)
$$

where $\phi(\omega, t)$ is the regular function; $R$ is the gas constant; $\omega=\rho / \rho_{c} ; x=\tau /|\Delta \rho|^{1 / \beta}$ is the scaling variable; $t=T / T_{c} ; \beta$ is the critical index.

We notice: there is a principal difference of our FEoS from FEoS $[4,15,18-20,22,23]$. We have included an additional component in $F_{\text {nreg }}(\rho, T)$. There is a special scale function, $a_{1}(x)$, in this additional component recommended in [35]. This principal modernization has allowed us to improve a FEoS structure and to meet the requirements [2].

The scaling functions $a_{0}(x)$ and $a_{1}(x)$ are calculated based on the following:

- a new representation of the scaling hypothesis [34,35];

- the Benedek hypothesis [36];

- the Berestov equation [2]. 


\begin{tabular}{llll}
\hline$i$ & $j=0$ & $j=1$ & $j=2$ \\
\hline 0 & 0 & 0 & 2.7270316121447 \\
1 & 0 & 0 & -2.1809170852935 \\
2 & 0 & 0 & 2.0181792856405 \\
3 & 0 & -1.6518073502083 & 1.951547138476 \\
4 & 0 & 3.2350970279452 & 5.8619678664433 \\
5 & 0 & 0.20326116428107 & -2.078078708984 \\
6 & -0.283648592739017 & -1.8506701543516 & -2.8017356060172 \\
-0.031673399139638 & 2.4978434896566 & 9.6202114551673 \\
-0.11731951178966 & -1.1649958742581 & -3.4762131583227 \\
9 & 0.41319373079189 & 2.8101660152324 & -0.81128861425157 \\
10 & -0.76560673765749 & -2.8011249735011 & 0.76666426064657 \\
11 & -0.6504913542378 & -2.5927427984863 & 0.45068490339798 \\
12 & 1.8085886445017 & 5.5787342684796 & -0.23925940579052 \\
13 & -1.042305956028 & -2.4850696471961 & -1.0249470330846 \\
14 & -0.81394497119275 & -2.3929979718019 & 0.75916486258433 \\
15 & 1.3280576071621 & 3.1197941258801 & 0 \\
16 & -0.48680310650006 & -0.9339859694002 & -0.22148907882357 \\
17 & -0.24347462543364 & -0.58562562879648 & 0.091451137589177 \\
18 & 0.32753066799216 & 0.66086037893756 & 0 \\
19 & -0.15406804632052 & -0.28654695547554 & -0.0086674736637731 \\
20 & 0.039124504337479 & 0.068175553922501 & 0.0018656951438862 \\
21 & -0.005346887409843 & -0.0087729629581014 & 0 \\
22 & 0.00031006718005802 & 0.00047873289794804 & $-2.5448089017224 \times 10^{-5}$ \\
\hline
\end{tabular}

Table 1: Coefficients $C_{i, j}$ of FEoS (1).

These functions are written the following form:

$$
\begin{gathered}
a_{0}(x)=-\frac{u_{0} k \gamma_{1} x_{0}^{2-\alpha}}{2 \alpha b^{2} \alpha_{1}(1-\varepsilon)}\left[\left(\varphi+\varphi_{1}\right)^{2-\alpha}-\varepsilon\left(\varphi+\varphi_{2}\right)^{2-\alpha}\right]+\frac{u_{0} x_{0}^{\gamma}}{2 k}\left(\varphi+\varphi_{3}\right)^{\gamma}+u_{0} C_{0}, \\
a_{1}(x)=-\frac{u_{1} k \gamma_{2} x_{0}^{2-\alpha+\Delta}}{2 \alpha b^{2} \alpha_{2}(1-\varepsilon)}\left[\left(\varphi+\varphi_{1}\right)^{2-\alpha+\Delta}-\varepsilon\left(\varphi+\varphi_{2}\right)^{2-\alpha+\Delta}\right]+\frac{u_{1} x_{0}^{\gamma+\Delta}}{2 k}\left(\varphi+\varphi_{3}\right)^{\gamma+\Delta}+u_{1} C_{1},
\end{gathered}
$$

where $\varepsilon_{0}=x_{1} / x_{2} ; \alpha_{1}=(2-\alpha)(1-\alpha) ; \gamma_{1}=\gamma(\gamma-1) ; \gamma_{2}=(\gamma+\Delta)(\gamma+\Delta-1) ; \alpha_{2}=(2-\alpha+$ $\Delta)(1-\alpha+\Delta) ; \varphi=x / x_{0} ; \varphi_{i}=x_{i} / x_{0}, i \in\{1,2,3\} ; b^{2}=(\gamma-2 \beta) /[\gamma(1-2 \beta)] ; k=\left[\left(b^{2}-1\right) / x_{0}\right]^{\beta}$; $\gamma$ is the critical index; $x_{0}, u_{0}$ and $u_{1}$ are the individual parameters; $Z_{c}=p_{c} /\left(R \rho_{c} T_{c}\right) \times 10^{3} ; C_{0}$ 


\begin{tabular}{lccl}
\hline$i$ & $j=3$ & $j=4$ & $j=5$ \\
\hline 0 & 4.4822485747539 & 2.3326430552399 & 1.8084657728776 \\
1 & -3.2256391060006 & -1.048810609669 & -0.93253183173191 \\
2 & -3.1430858007921 & -7.4166502306154 & -4.1562405172991 \\
3 & 7.6516533027528 & 6.8055769267176 & 3.9404591009914 \\
4 & 6.1186676232535 & 4.7373818378476 & 0.74144113878428 \\
5 & -8.02979776049146 & -8.2325100770624 & -2.1726725072028 \\
-0.081651952400293 & 1.4887727093593 & 0.93023543296788 \\
7 & 8.3881567003335 & 2.5108162887711 & -0.13087346335537 \\
8 & -4.0590171371799 & -1.0892472187001 & 0 \\
9 & -0.10375999144993 & -0.27775442560302 & 0 \\
10 & 1.129529996898 & 0.093168194589203 & 0 \\
$11-0.54376508852754$ & 0.1513360277963 & 0 \\
12 & 0.0086451032482461 & -0.082484913633882 & 0 \\
13 & 0.0018217794283432 & 0.012151299548948 & 0 \\
\hline
\end{tabular}

Table 2: Coefficients $C_{i, j}$ of FEoS (1).

and $C_{1}$ are the constant coefficients which value is found from the equations

$$
\begin{gathered}
\left.(\delta+1) a_{0}\right|_{\varphi=-1}+\left.\frac{x_{0}}{\beta} a_{0}^{\prime}\right|_{\varphi=-1}=0, \\
\left.\left(\delta+1+\frac{\Delta}{\beta}\right) a_{1}\right|_{\varphi=-1}+\left.\frac{x_{0}}{\beta} a^{\prime}\right|_{\varphi=-1}=0 .
\end{gathered}
$$

We have selected the regular component (1) in the form [19]:

$$
\begin{gathered}
F_{\mathrm{reg}}(\rho, T)=F^{0}(\rho, T)+R T \omega y_{2}+R T \omega\left(Z_{c}-0.2\right) y_{6}+R T \omega D_{3}\left(y_{4}-y_{6}\right) \\
+R T \omega \tau_{1}\left[D_{1}(\omega-3)+D_{2}\left(\omega^{2}-2 \omega\right)\right]+R T \omega \sum_{i=0}^{22} \sum_{j=0}^{20}\left(C_{i, j} \tau_{1}^{j} \Delta \rho^{i}\right),
\end{gathered}
$$

where $F^{0}(\rho, T)$ is the ideal gas component of $F(\rho, T) ; \tau_{1}=T_{c} / T-1$; functions $y_{2}, y_{4}, y_{6}$ have the following form: $y_{2}=-7.7 / 6+2.9 / 6 \Delta \rho-1.1 / 6 \Delta \rho^{2}+0.05 \Delta \rho^{3}, y_{4}=5-4 \Delta \rho+3 \Delta \rho^{2}-$ $2 \Delta \rho^{3}+\Delta \rho^{4}, y_{6}=4-3 \Delta \rho+2 \Delta \rho^{2}-\Delta \rho^{3}+\Delta \rho^{5}$.

We have calculated $\varphi_{1}, \varphi_{2}, \varphi_{3}$ values according to the method detailed in [15]. It let us got $\varphi_{1}=2.80722347, \varphi_{2}=14.4717304, \varphi_{3}=5.73246825$. 


\begin{tabular}{lccc}
\hline$i$ & $j=6$ & $j=7$ & $j=8$ \\
\hline 0 & 1.9420563200621 & 3.2452464931065 & -8.2394067009885 \\
1 & -1.0346405643285 & -0.97015956031712 & -0.011163693637208 \\
2 & -0.9441395672871 & $0.298 \quad 85960268675$ & 0.039252086979538 \\
3 & 1.2041159466534 & 0 & 0 \\
4 & -0.32749951226419 & 0 & 0 \\
\hline$i$ & $j=9$ & $j=10$ & $j=11$ \\
\hline 0 & -18.746448404883 & 51.077633966366 & 68.64532945291 \\
1 & 0.54107255079912 & 0 & 0 \\
2 & -0.17804461988026 & 0 & 0 \\
\hline$i$ & $j=12$ & $j=13$ & $j=14$ \\
\hline 0 & -182.0473713271 & -144.87007187434 & 383.40615547806 \\
\hline$i$ & $j=15$ & $j=16$ & $j=17$ \\
\hline 0 & 174.01764151555 & -472.41883833036 & -110.34717301813 \\
\hline$i$ & $j=18$ & $j=19$ & $j=20$ \\
\hline 0 & 314.55286984435 & 28.506239206301 & -87.384487306415 \\
\hline
\end{tabular}

Table 3: Coefficients $C_{i, j}$ of FEoS (1).

We have chosen the crossover function in accordance with the recommendations [7]:

$$
\phi(\omega, t)=\phi_{0}(\omega) \phi_{1}(t), \quad \phi_{0}(\omega)=\left[(1-\omega)^{m}-1\right]^{2}, \quad \phi_{1}(t)=1 / t^{2},
$$

where $m \in N$.

We have tested FEoS (1) with components (2)-(4) and (7) on the example describing the equilibrium properties of argon [37-54].

\section{FEOS OF ARGON}

Select the ideally-gas component of $\arg g F^{0}(T, \rho)$ according to the recommendations of [9]:

$$
F^{0}(\rho, T)=R T\left(\ln \omega+a_{1}^{0}+a_{2}^{0} t^{-1}-1.5 \ln t\right)
$$

where $a_{1}^{0}=58.31666243$ and $a_{2}^{0}=524.94651164$. 


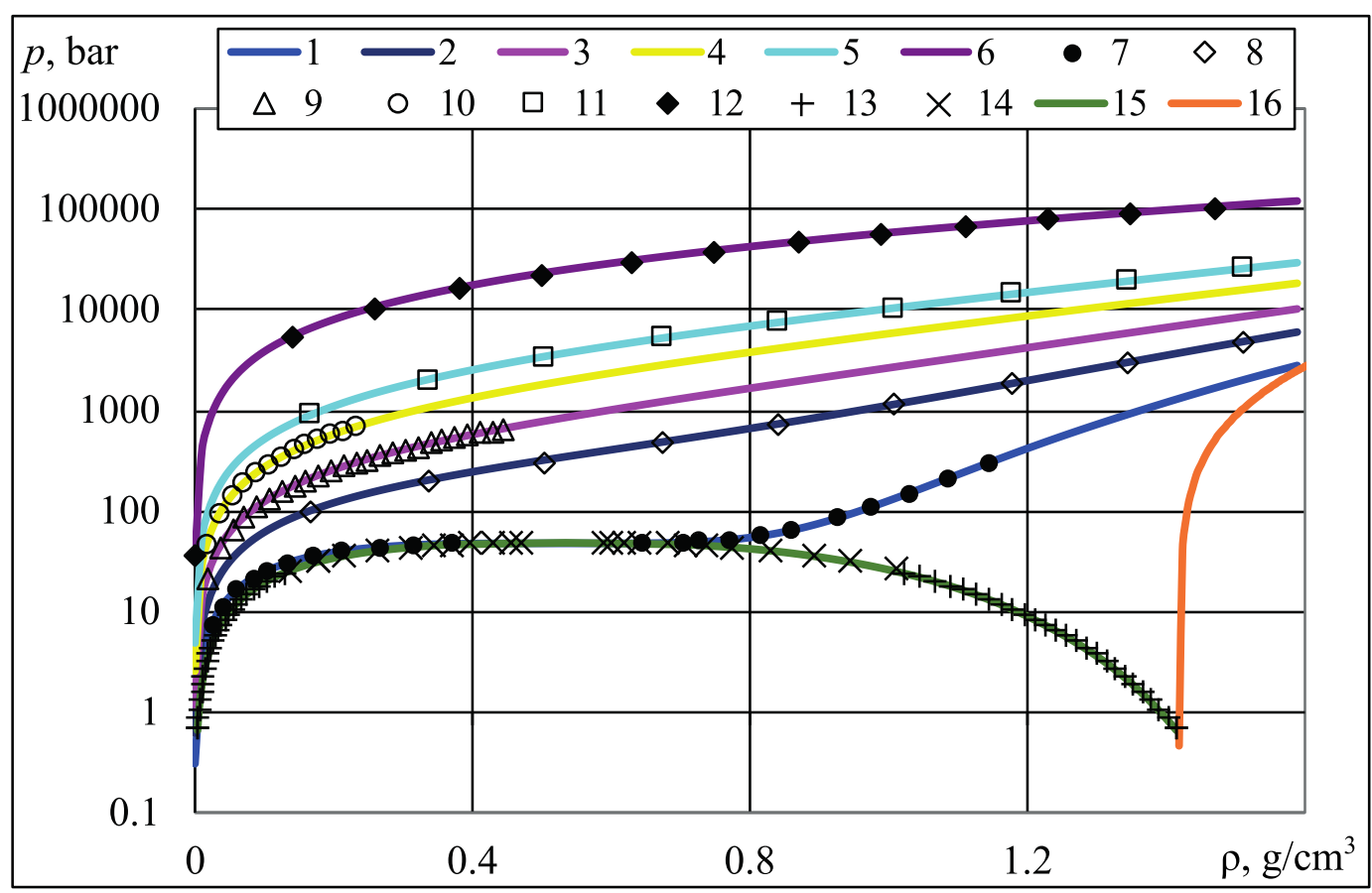

Figure 1: Isotherms, the liquid-gas coexistence curve and the melting line of argon. Isotherms calculated using FEoS (1): 1—critical isotherm; 2-300 K; 3-573.15 K; 4-1223.15 K; 5-2300 K; 6-17000 K. Experimental data: 7-150.65 K [42]; 8-300 K [3]; 11-2300 K [3]. Tabulated data: 9

- 573.15 K [43]; 10—1223.15 K [43]; 12—17000 K [9]; 13—tabulated data on the density at the saturation line [9]; 14 - experimental data on the density of a saturated liquid and saturated vapor [49]; 15-data on the density at the saturation line calculated by FEoS (1); 16 - data on the density at the melting line [9].

We have calculated expressions for compressibility $Z$ on the basis of FEoS (1) with components (2)-(4) and (7):

$$
\begin{gathered}
Z(\rho, T)=1+y_{1} \omega^{2}+y_{2} \omega+D_{3}\left(y_{3} \omega^{2}+y_{4} \omega-y_{5} \omega^{2}-y_{6} \omega\right)+\left(y_{5} \omega^{2}+y_{6} \omega\right)\left(Z_{c}-0.2\right) \\
+\omega \sum_{i=0}^{22} \sum_{j=0}^{20} C_{i, j} \tau_{1}{ }^{j} \Delta \rho^{i-1}(i \omega+\Delta \rho)+D_{1} \omega \tau_{1}(2 \omega-3)+D_{2} \omega^{2} \tau_{1}(3 \omega-4) \\
\quad+Z_{c} \omega|\Delta \rho|^{\delta} \phi_{1}(t) t\left(\phi_{0}(\omega) \operatorname{sign}(\Delta \rho) h_{0}(x)+\phi^{\prime}{ }_{0}(\omega)|\Delta \rho| a_{0}(x)\right) \\
+Z_{c} \omega|\Delta \rho|^{\delta+\frac{\Delta}{\beta}} \phi_{1}(t) t\left(\phi_{0}(\omega) \operatorname{sign}(\Delta \rho) h_{1}(x)+\phi^{\prime}{ }_{0}(\omega)|\Delta \rho| a_{1}(x)\right)
\end{gathered}
$$

where $y_{2 i-1}=y_{2 i}^{\prime}(\omega)(i \in\{1,2,3\}) ; h_{n}(x)$ are scale functions of chemical potential [27]:

$$
h_{0}(x)=(\delta+1) a_{0}(x)-\frac{x}{\beta} a_{0}^{\prime}(x), \quad h_{1}(x)=\left(\delta+1+\frac{\Delta}{\beta}\right) a_{1}(x)-\frac{x}{\beta} a_{1}^{\prime}(x) .
$$




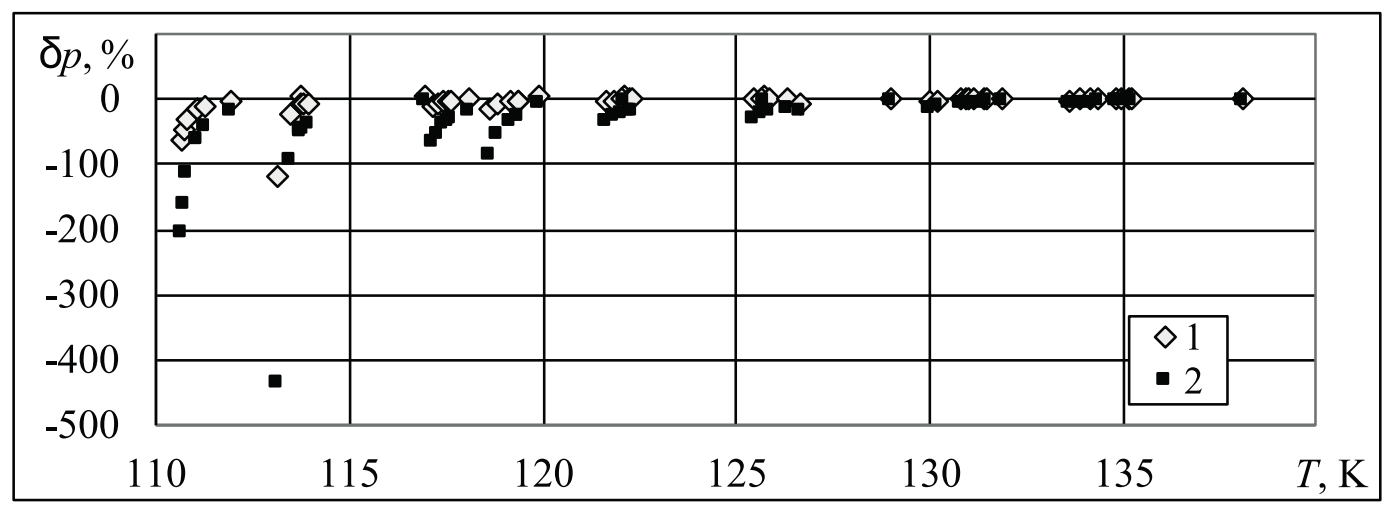

Figure 2: Relative deviations $\delta p=\left(p_{\exp }-p_{\text {cal }}\right) / p_{\exp } 100 \%$ corresponded to $p_{\text {cal values calculated with the help }}$ of FEoS (1) and FEoS [9] in the metastable states of argon: ( $\left.p_{\exp }, \rho_{\exp }, T_{\exp }\right)$ data are taken from [45] over isochoric lines $1231.9,1210.9,1180.2,1165.6,1140.9,1099.8,1050.8$ and $1010.7 \mathrm{~kg} / \mathrm{m}^{3} ; 1-p_{\text {cal values }}$ calculated with the help of FEoS $(1) ; 2-p_{\text {cal }}$ values calculated with the help of FEoS [9]. On each of the isochors, two experimental points corresponding to large values of the pressure are located in the single-phase range, the rest of experimental points are located in the metastable range.

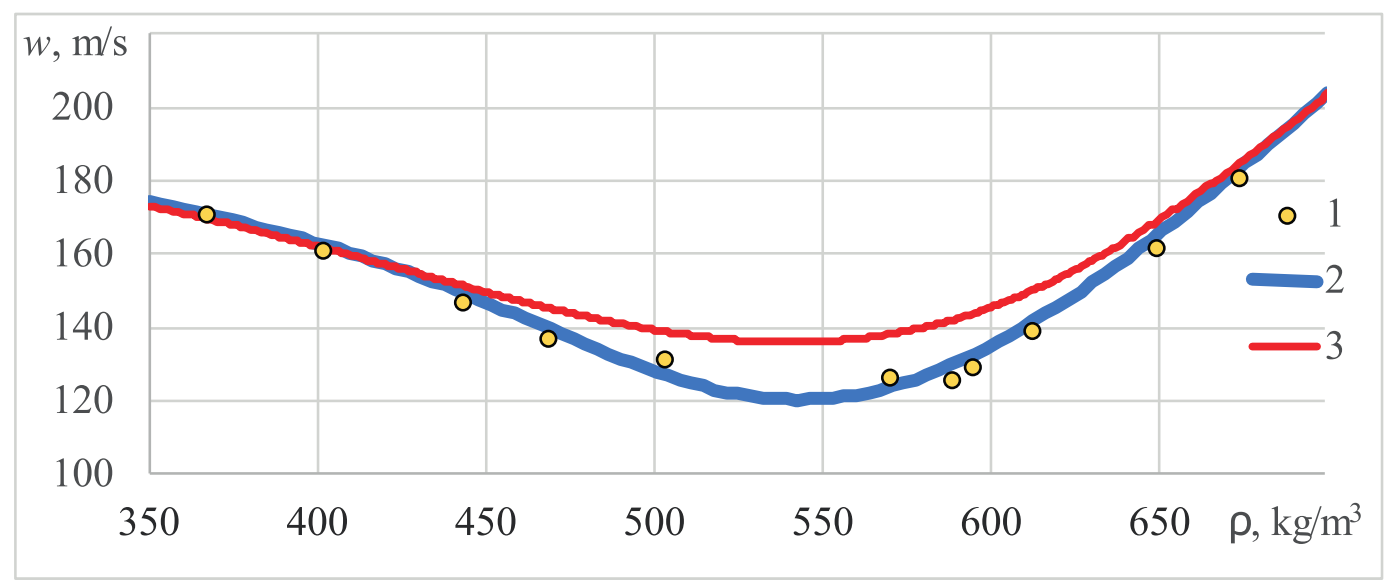

Figure 3: Argon sound speed at $150.8 \mathrm{~K}$ isotherm: 1-experimental data [46]; 2-calculation by FEoS (1); 3calculation by FEoS [9].

Coefficients and parameters of FEoS (1) with components (2)-(4) and (7), (8) were determined on the basis of an array of experimental data [37-54] among them: $T_{c}=150.66 \mathrm{~K}$, $p_{c}=4.8634 \mathrm{MPa}, \rho_{c}=535.1 \mathrm{~kg} / \mathrm{m}^{3}, R=0.20813332 \mathrm{~J} /(\mathrm{g} \mathrm{K}), u_{0} Z_{c}=4.54936419, u_{1} Z_{c}=$ $0.0524296231552, \alpha=0.11, \beta=0.3255, \gamma=1.239, \delta=4.806, \Delta=0.51, m=3, D_{1}=$ $0.52854169554602, D_{2}=0.87466821897252, D_{3}=-7.9131735557194 \times 10^{-3}$ and $x_{0}=$ 0.31122037639966 . The values of coefficients $C_{i, j}$ are presented in tables 1,2 and 3. 


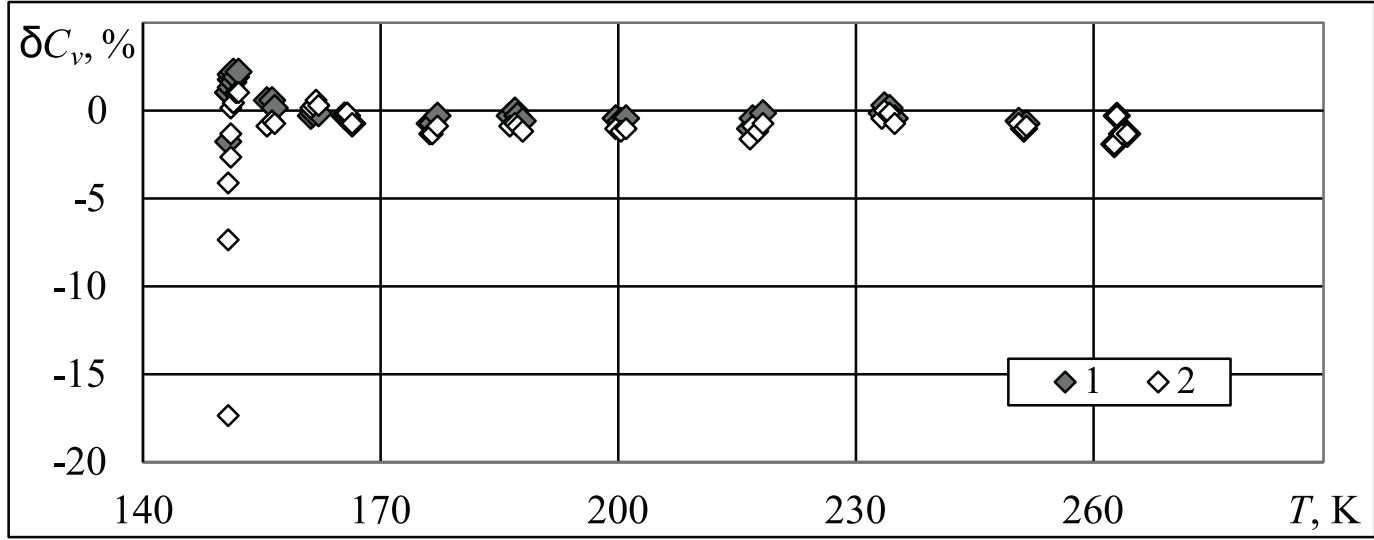

Figure 4: Relative deviations $\delta C V=(C V$,exp $-C V$,cal $) / C V$,exp $100 \%$; $p_{\text {cal }} C_{V}$,cal corresponded to values calculated with the help of (1) in the single phase range; $C V$,exp corresponded to data [48] over isochoric line $473.6 \mathrm{~kg} / \mathrm{m}^{3} ; 1-C V$ calculated with the help of FEoS (1); $C V$ calculated with the help of FEoS [9].

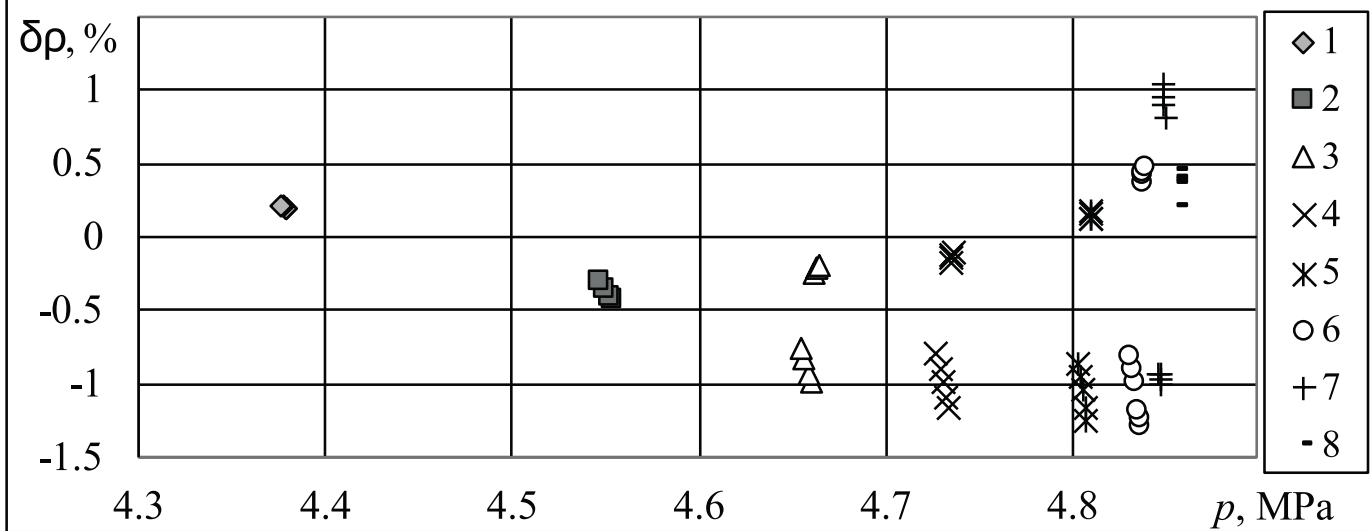

Figure 5: Relative divergence values of density $\delta \rho=\left(\rho_{\exp }-\rho_{\text {calc }}\right) / \rho_{\exp } 100 \%$ calculated as per the equations pre-sented in this study as compared with the experimental data [44]. Isothermal lines: 1-148.007 K; 2 - 149.006 $\mathrm{K} ; 3-149.598 \mathrm{~K} ; 4-149.983 \mathrm{~K} ; 5-150.372 \mathrm{~K} ; 6-150.52 \mathrm{~K} ; 7-150.579 \mathrm{~K} ; 8-150.621 \mathrm{~K}$.

Based on compressibility $Z$ (10), we have calculated the thermal surface of argon (figure 1). As one can see, FEoS (1) transmits the thermal surface of argon in the temperature range from the saturation line and the melting line to $2300 \mathrm{~K}$ and it can be extrapolated by temperature up to $17000 \mathrm{~K}$ and by pressure up to $12 \mathrm{GPa}$. FEoS (1) describes the experimental $(p, \rho, T)$-data in the metastable range [45], experimental data about $C_{V}$ [48] and about the speed of sound $w$ [46] in the vicinity of the critical point with less uncertainty than FEoS NIST [9] (figures 2, 3 and 4). Note that when searching for the coefficients of FEoS (1), experimental data [45,46] were not used. The FEoS (1) represents experimental $(p, \rho, T)$-data [44] within the range of the experimental error (figure 5). Experimental data on $C_{V}[48,50]$ are transmitted within the experimental error (figure 6) in a wide range of state parameters including the vicinity of the critical point. 


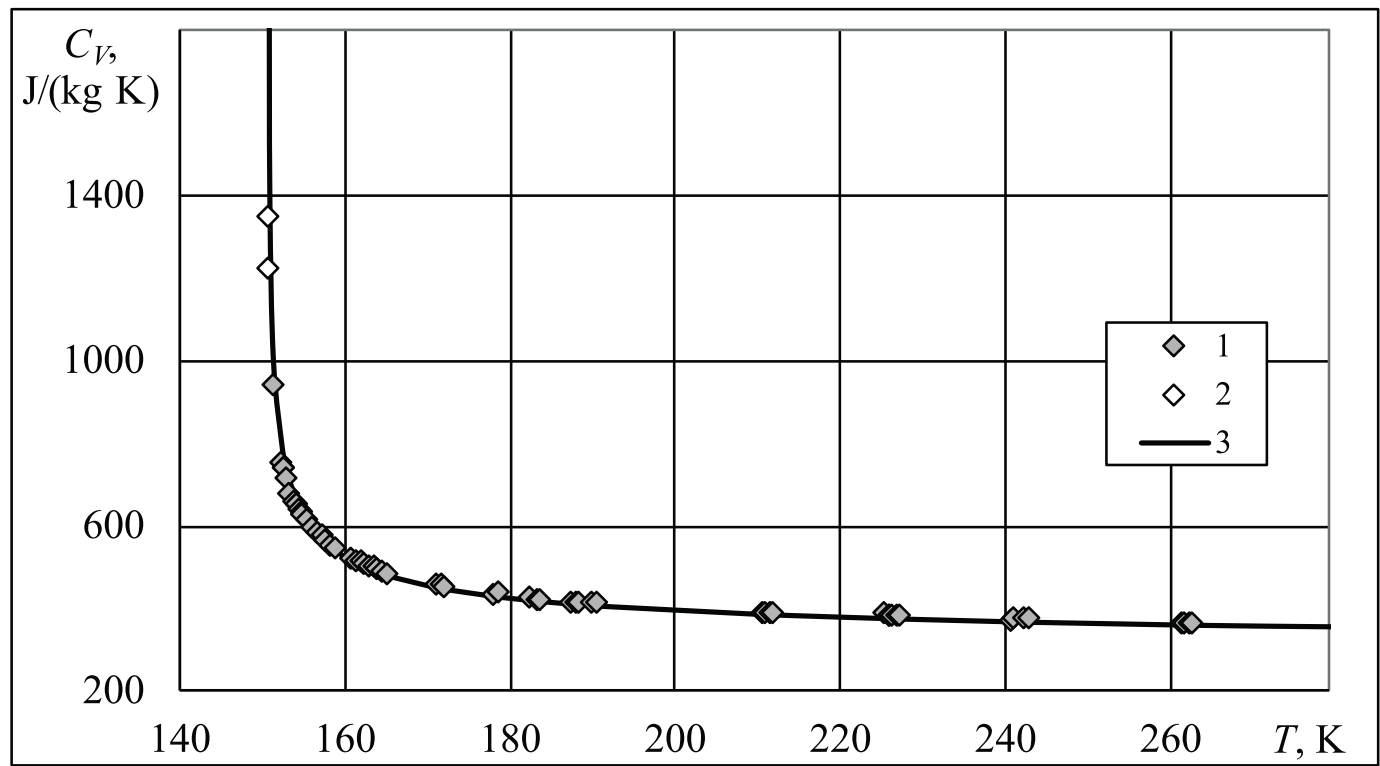

Figure 6: Behavior of the isochoric heat capacity of argon in a single-phase region: 1 -experimental points [48] over the isochor of $531 \mathrm{~kg} / \mathrm{m}^{3} ; 2$-experimental points [50] over the isochor $530 \mathrm{~kg} / \mathrm{m}^{3} ; 3-C_{V}$ calculated with a help of the FEoS (1) over isochor $530.5 \mathrm{~kg} / \mathrm{m}^{3}$.

\section{CONCLUSIONS}

On the basis of a new representation of the scaling hypothesis $[34,35]$ and the Berestov equation [2], FEoS (1) was developed. This FEoS primarily works satisfactorily in a wide range of pressures and temperatures including the critical range and the range of high temperatures and pressures. FEoS (1) with components (2)-(4) and (7) has the properties of the virial series in the regular part of the thermodynamic surface as well as the properties of the Berestov equation in the critical range.

Argon FEoS (1) can be used to calculate the equilibrium properties in various technology processes. We have analyzed properties calculated with the help of FEoS (1) in the vicinity of the critical point. Our values significantly exceeds the accuracy of the data generated with the help of known FeoSs and known crossover EoSs $[6,9,12,17]$.

The proposed method of constructing FEoS can be recommended for developing EoSs of substances, which have reliable experimental data, for example, it carbon dioxide and sulfur hexafluoride.

Calculated values of properties from the FEoS (1) to verify computer code are $T=400 \mathrm{~K}$, $\rho=1000 \mathrm{~kg} / \mathrm{m}^{3}, p(T, \rho)=168974.25 \mathrm{kPa}, C_{V}(T, \rho)=0.3920699 \mathrm{~J} /(\mathrm{g} \mathrm{K})$.

Acknowledgments: The paper is based on the proceedings of the XXXIV International Conference on Interaction of Intense Energy Fluxes with Matter, Elbrus, the Kabardino-Balkar Republic of the Russian Federation, March 1 to 6, 2019. 


\section{REFERENCES}

[1] P. Schofield, J. D. Litster, and J. T. Ho, "Correlation between critical coefficients and critical exponents", Phys. Rev. Lett., 23(19), 1098-1102 (1969).

[2] A. T. Berestov, "Equation of state in the critical region with inclusion of non-asymptotic terms", Zh. Eksp. Teor. Fiz., 72(1), 348-353 (1977).

[3] C. Ronchi, "Extrapolated equation of state for rare gases at high temperatures and densities", $J$. Nucl. Mater., 96(3), 314-328 (1981).

[4] V. A. Rykov and G. B. Varfolomeeva, "Method of determining a structural form of the free energy satisfying the requirements of the scaling hypothesis", J. Eng. Phys. Thermophys., 48(3), 341-345 (1985).

[5] V. A. Rykov, "Method of constructing a single equation of state satisfying the requirements of the scaling hypothesis", J. Eng. Phys. Thermophys., 48(4), 476-481 (1985).

[6] R. B. Stewart and R. T. Jacobsen, "Thermodynamic properties of argon from the triple point to 1200 K with pressures to 1000 MPa", J. Phys. Chem. Ref. Data, 18(2), 639-798 (1989).

[7] A. D. Kozlov, V. F. Lysenkov, P. V. Popov, and V. A. Rykov, "Unified nonanalytical equation of state for freon-218", J. Eng. Phys. Thermophys., 62(6), 611-617 (1992).

[8] V. F. Lysenkov, A. D. Kozlov, P. V. Popov, and M. V. Yakovleva, "Nonanalytical unified equation of state of freezant R23", J. Eng. Phys. Thermophys., 66(3), 286-294 (1994).

[9] Ch. Tegeler, R. Span, and W. Wagner, "A new equation of state for argon covering the fluid region for temperatures from the melting line to $700 \mathrm{~K}$ at pressures up to $1000 \mathrm{MPa}$ ", J. Phys. Chem. Ref. Data, 28(3), 779-850 (1999).

[10] L. Sun, S. B. Kiselev, and J. F. Ely, "Multiparameter crossover equation of state: Generalized algorithm and application to carbon dioxide", Fluid Phase Equilib., 233(2), 204-219 (2005).

[11] I. V. Kudryavtseva, V. A. Rykov, and S. Rykov, "Asymmetric unified equation of state for R134a", Journal of IAR, 2, 36-39 (2008).

[12] A. Rizi and A. Abbaci, "A thermodynamic equation of state for the critical region of argon", J. Mol. Liq., 171, 64-70 (2012).

[13] V. I. Mazhukin, A. V. Shapranov, O. N. Koroleva, and A. V. Rudenko, "Molecular dynamics simulation of critical point parameters for silicon", Math. Montis., 31, 64-77 (2014).

[14] P. P. Bezverkhy and V. G. Martynets, "Calculation of thermodynamic properties of $\mathrm{CO}_{2}$ using the combined thermal equation of state with a small number of adjustable parameters", High Temp.High Pressures, 45(2), 145-154 (2016).

[15] S. V. Rykov, I. V. Kudryavtseva, V. A. Rykov, M. I. Poltoratskiy, and A. V. Sverdlov, "Equation of state for refrigerant R32", Kholod. Tekh., 11, 34-37 (2016).

[16] I. V. Kudryavtseva and S. V. Rykov, "A nonparametric scaling equation of state, developed on the basis of the Migdal's phenomenological theory and Benedek's hypothesis", Russ. J. Phys. Chem. A, 90(7), 1493-1495 (2016).

[17] A. B. Kaplun and A. B. Meshalkin, "Simple fundamental equation of state for liquid, gas, and fluid of argon, nitrogen, and carbon dioxide", Thermophys. Aeromech., 55, 513-522 (2017).

[18] M. I. Poltoratskiy, Method of constructing a fundamental equation of state and thermodynamic tables for hexafluoropropane (R236ea), PhD thesis, Saint-Petersburg: ITMO University, (2018).

[19] I. V. Kudryavtseva, V. A. Rykov, S. V. Rykov, and E. E. Ustyuzhanin, "A new variant of a scaling hypothesis and a fundamental equation of state based on it", J. Phys.: Conf. Ser., 946, 012118 (2018).

[20] S. V. Rykov, I. V. Kudryavtseva, V. A. Rykov, and A. V.Zaitsev, "Methods for calculating equilibrium properties of pure substances, considering the critical point features", J. Phys.: Conf. Ser., 1128, 012106 (2018).

[21] O. N. Koroleva, M. M. Demin, V. I. Mazhukin, and A. V. Mazhukin, "Modeling of thermal conductivity of Si in the range from the normal to near-critical conditions", Math. Montis., 45, 85-94 (2019).

[22] I. V. Kudryavtseva, V. A. Rykov, and S. V. Rykov, "The method for constructing the fundamental equation of state for $\mathrm{SF}_{6}$ ", J. Phys.: Conf. Ser., 1385, 012009 (2019). 
[23] V.A. Rykov, S. V. Rykov, and A. V. Sverdlov, "Fundamental equation of state for R1234yf", $J$. Phys.: Conf. Ser., 1385, 012013 (2019).

[24] S. V. Rykov, I. V. Kudryavtseva, V. A. Rykov, and E. E. Ustyuzhanin, "Scaling Migdal model and a nonparametric equation of state for argon", J. Phys.: Conf. Ser., 1147, 012018 (2019).

[25] V. E. Fortov, K. V. Khishchenko, P. R. Levashov, and I. V. Lomonosov, "Wide-range multi-phase equations of state for metals", Nucl. Instr. Meth. Phys. Res. A, 415, 604-608 (1998).

[26] K. V. Khishchenko, "Equations of state for two alkali metals at high temperatures", J. Phys.: Conf. Ser., 98, 032023 (2008).

[27] I. V. Lomonosov and S. V. Fortova, "Wide-range semiempirical equations of state of matter for numerical simulation on high-energy processes", High Temp., 55, 585-610 (2017).

[28] K. V. Khishchenko, "Equation of state of sodium for modeling of shock-wave processes at high pressures", Math. Montis., 40, 140-147 (2017).

[29] K. V. Khishchenko, "Equation of state for lithium in shock waves", Math. Montis., 41, 91-98 (2018).

[30] K. K. Maevskii, "Thermodynamic parameters of lithium deuteride in pressure range 5-1000 gigapascals", Math. Montis., 41, 123-130 (2018).

[31] K. V. Khishchenko, "Equation of state for magnesium hydride under conditions of shock loading", Math. Montis., 43, 70-77 (2018).

[32] K. V. Khishchenko, "Equations of state for rubidium and cesium at high pressures in shock waves", J. Phys.: Conf. Ser., 1147, 012001 (2019).

[33] Sh. Ma, Modern Theory of Critical Phenomena, New York, NY: Roudedge, (1976).

[34] S. V. Rykov, I. V. Kudryavtsev, and V. A. Rykov, "The physical basis of the method of pseudocritical points", Scientific and Technical Bulletin of Povolzhie, 2, 44-47 (2014).

[35] S. V. Rykov, "Fundamental equation of state taking into account liquid asymmetry", Scientific and Technical Bulletin of Povolzhie, 1, 33-36 (2014).

[36] G. B. Benedek, in: Polarization Matiere et Rayonnement, Livre de Jubile en l'Honneur du Professeur A. Kastler Paris: Presses Universitaires de Paris, 71 (1968).

[37] A. Michels, J. M. Levelt, and G. J. Wolkers, "Thermodynamic properties of argon at temperatures between $0{ }^{\circ} \mathrm{C}$ and $-140{ }^{\circ} \mathrm{C}$ and at densities up to 640 amagat (pressures up to $1050 \mathrm{~atm}$.)", Physica, 24(6), 769-794 (1958).

[38] S. L. Robertson, S. E. Babb, and G. J. Scott, "Isotherms of argon to 10000 bars and $400{ }^{\circ} \mathrm{C}$ ", $J$. Chem. Phys., 50(5), 2160-2166 (1969).

[39] W. B. Streett and L. A. K. Staveley, "Experimental study of the equation of state of liquid argon", J. Chem. Phys., 50(6), 2302-2307 (1969).

[40] A. Van Itterbeek and O. Verbeke, "Density of liquid nitrogen and argon as a function of pressure and temperature", Physica, 26(11), 931-938 (1960).

[41] E. Whalley, Y. Lupien, and W. G. Schneider, "The compressibility of gases: VII. Argon in the temperature range $0-600{ }^{\circ} \mathrm{C}$ and the pressure range 10-80 atmospheres", Can. J. Chem., 31(8), $722-$ 733 (1953).

[42] A. Michels, J. M. Levelt, and W. D. Graaff, "Compressibility isotherms of argon at temperatures between $-25^{\circ} \mathrm{C}$ and $-155^{\circ} \mathrm{C}$, and at densities up to 640 amagat (pressures up to 1050 atmospheres)", Physica, 24(6), 659-671 (1958).

[43] A. Lecocq, "Determination experimentale des equations d'etat de l'argon jusqua $1000{ }^{\circ} \mathrm{C}$ et $1000 \mathrm{~kg} / \mathrm{m}^{3}$ ", J. Recherches Centre National Recherche Scientific, 55, 55-82 (1960).

[44] R. Gilgen, R. Kleinrahm, and W. Wagner, "Measurement and correlation of the (pressure, density, temperature) relation of argon I. The homogeneous gas and liquid regions in the temperature range from $90 \mathrm{~K}$ to $340 \mathrm{~K}$ at pressures up to $12 \mathrm{MPa}$, J. Chem. Thermodyn., 26(4), 383-398 (1994).

[45] V. P. Skripov, E. N. Sinitsyn, P. A. Pavlov, G. V. Ermakov, G. N. Muratov, N. V. Bulanov, and V. Baidakov, Teplofizicheskiye Svoystva Zhidkostey v Metastabil'nom Sostoyanii (Thermophysical Properties of Liquids in a Metastable State), Moscow: Atomizdat, (1980).

[46] J. Thoen, E. Vangeel, and W. V. Dael, "Experimental investigation of the sound velocity in the critical region of argon", Physica, 52(2), 205-224 (1971).

[47] C. Gladun, "The specific heat of liquid argon", Cryogenics, 11(3), 205-209 (1971). 
[48] M. A. Anisimov, B. A. Koval'chuk, V. A. Rabinovich, and V. A. Smirnov, Teplofizicheskiye Svoystva Veshchestv $i$ Materialov (Thermophysical properties of substances and materials), 12, 85-106 (1978).

[49] R. Gilgen, R. Kleinrahm, and W. Wagner, "Measurement and correlation of the (pressure, density, temperature) relation of argon II. Saturated-liquid and saturated-vapour densities and vapour pressures along the entire coexistence curve", J. Chem. Thermodyn., 26(4), 399-413 (1994).

[50] A. V. Voronel' and Yu. R. Chashkin, "Specific heat $C_{v}$ of argon as a function of density near the critical point", Sov. Phys. JETP, 24(2), 263-267 (1967).

[51] J. Klimeck, R. Kleinrahm, and W. Wagner, "An accurate single-sinker densimeter and measurements of the $(p, \rho, T)$ relation of argon and nitrogen in the temperature range from (235 to 520) $\mathrm{K}$ at pressures up to $30 \mathrm{MPa}$ ", J. Chem. Thermodyn., 30(12), 1571-1588 (1998).

[52] A. V. Voronel', V. G. Snigirev, and Yu. R. Chashkin, "Behavior of the specific heat $C_{v}$ of pure substances near the critical point", Sov. Phys. JETP, 21(3), 653-655 (1965).

[53] A. Michels, H. Wijker, and H. Wijker, "Isotherms of argon between $0{ }^{\circ} \mathrm{C}$ and $150{ }^{\circ} \mathrm{C}$ and pressures up to 2900 atmospheres", Physica, 15(7), 627-633 (1949).

[54] R. K. Crawford and W.B. Daniels, "Equation-of-state measurements in compressed argon", $J$. Chem. Phys., 50(8), 3171-3183 (1969).

Received November 15, 2019 